BMC

Evolutionary Biology

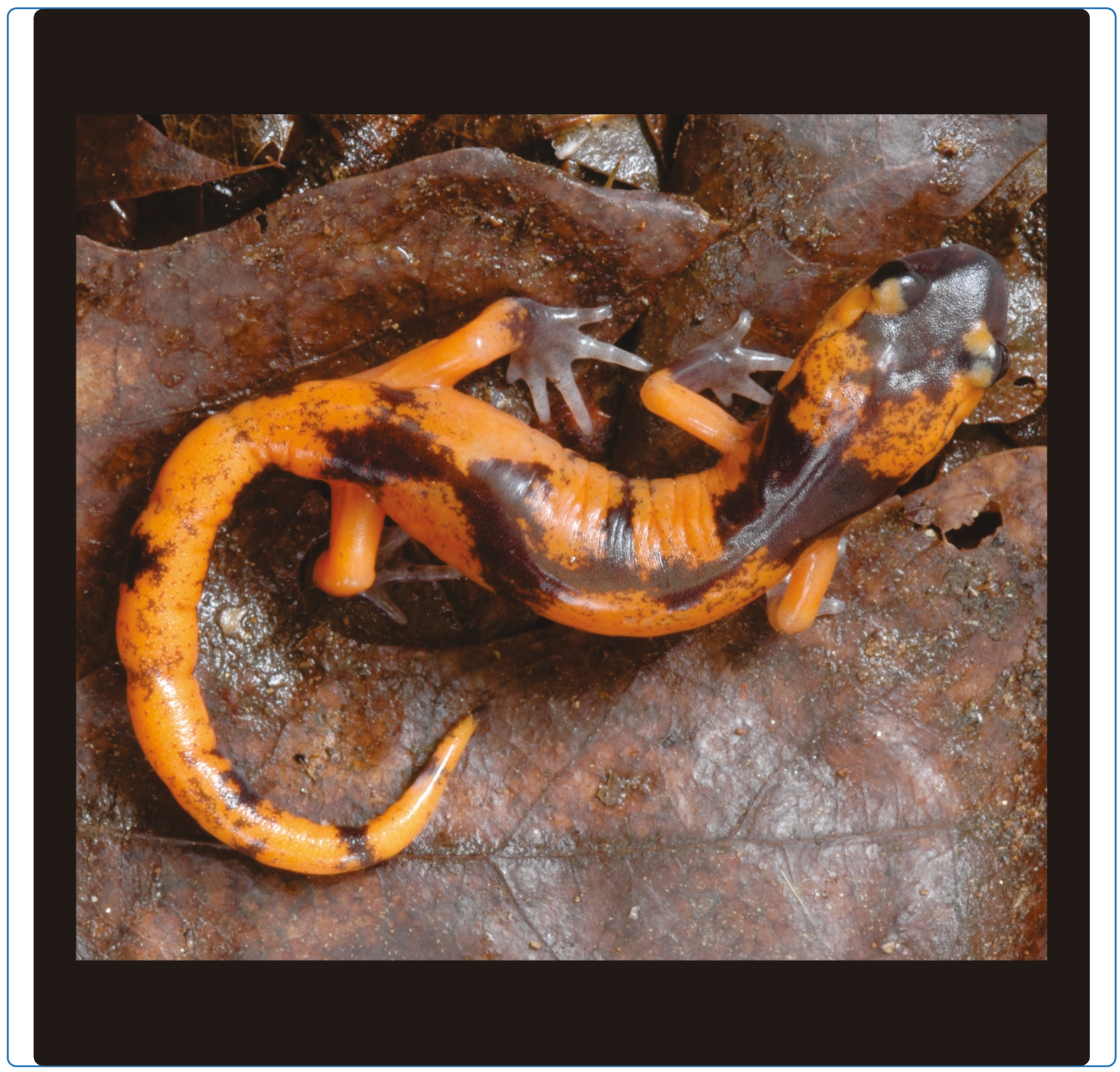

Asymmetric reproductive isolation between terminal forms of the salamander ring species Ensatina eschscholtzii revealed by fine-scale genetic analysis of a hybrid zone

Devitt et al. 


\title{
Asymmetric reproductive isolation between terminal forms of the salamander ring species Ensatina eschscholtzii revealed by fine-scale genetic analysis of a hybrid zone
}

Thomas J Devitt ${ }^{1,3^{*}}$, Stuart JE Baird ${ }^{2}$ and Craig Moritz ${ }^{1}$

\begin{abstract}
Background: Ring species, exemplified by salamanders of the Ensatina eschscholtzii complex, represent a special window into the speciation process because they allow the history of species formation to be traced back in time through the geographically differentiated forms connecting the two terminal forms of the ring. Of particular interest is the nature and extent of reproductive isolation between the geographically terminal forms, in this case E. e. eschscholtzii and E. e. klauberi. Previous studies have documented infrequent hybridization at the end of the ring. Here, we report the first fine-scale genetic analysis of a hybrid zone between the terminal forms in southern California using individual-based Bayesian analyses of multilocus genetic data to estimate levels and direction of hybridization and maximum-likelihood analysis of linkage disequilibrium and cline shape to make inferences about migration and selection in the hybrid zone.

Results: The center of the hybrid zone has a high proportion of hybrids, about half of which were classified as F1s. Clines are narrow with respect to dispersal, and there are significant deviations from Hardy-Weinberg equilibrium as well as nonrandom associations (linkage disequilibria) between alleles characteristic of each parental type. There is cytonuclear discordance, both in terms of introgression and the geographic position of mitochondrial versus nuclear clines. Genetic disequilibrium is concentrated on the eschscholtzii side of the zone. Nearly all hybrids possess klauberi mtDNA, indicating that most hybrids are formed from female klauberi mating with male eschscholtzii or male hybrids (but not vice versa).

Conclusions: Our results are consistent with a tension zone trapped at an ecotone, with gene combinations characteristic of klauberi showing up on the eschscholtzii side of the zone due to asymmetric hybridization. We suggest that the observed asymmetry is best explained by increased discriminatory power of eschscholtzii females, or asymmetric postzygotic isolation. The relatively high frequency of hybrids, particularly F1s, contrasts with other contacts between the terminal forms, and with other contacts between other divergent Ensatina lineages,

highlighting the diverse outcomes of secondary contact within a single species complex.
\end{abstract}

\section{Background}

Theoretical models predict that speciation can occur among continuously distributed populations isolated by distance alone, particularly when subject to divergent ecological selection [1-3]. Ring species - cases where two sympatric forms are connected by a chain of intergrading populations encircling a central geographic

\footnotetext{
* Correspondence: tommydevitt@gmail.com

'Museum of Vertebrate Zoology and Department of Integrative Biology, 3101 Valley Life Sciences Building, University of California, Berkeley, CA, USA 94720-3160

Full list of author information is available at the end of the article
}

barrier [4-7] - present the ideal opportunity to test this prediction $[8,9]$, especially when the interacting lineages are ecologically divergent. Of particular interest in such cases is the nature and extent of reproductive isolation between the terminal forms where they are sympatric $[1,10,11]$. The Ensatina eschscholtzii plethodontid salamander complex of western North America is a famous example of a ring species [5,12-15]. These salamanders inhabit mesic, forested environments in Pacific western North America, and in California form a geographic ring around the arid Central Valley (Figure 1). In his detailed analysis of geographic variation and speciation
C Biomed Central

(c) 2011 Devitt et al; licensee BioMed Central Ltd. This is an Open Access article distributed under the terms of the Creative Commons Attribution License (http://creativecommons.org/licenses/by/2.0), which permits unrestricted use, distribution, and reproduction in any medium, provided the original work is properly cited. 


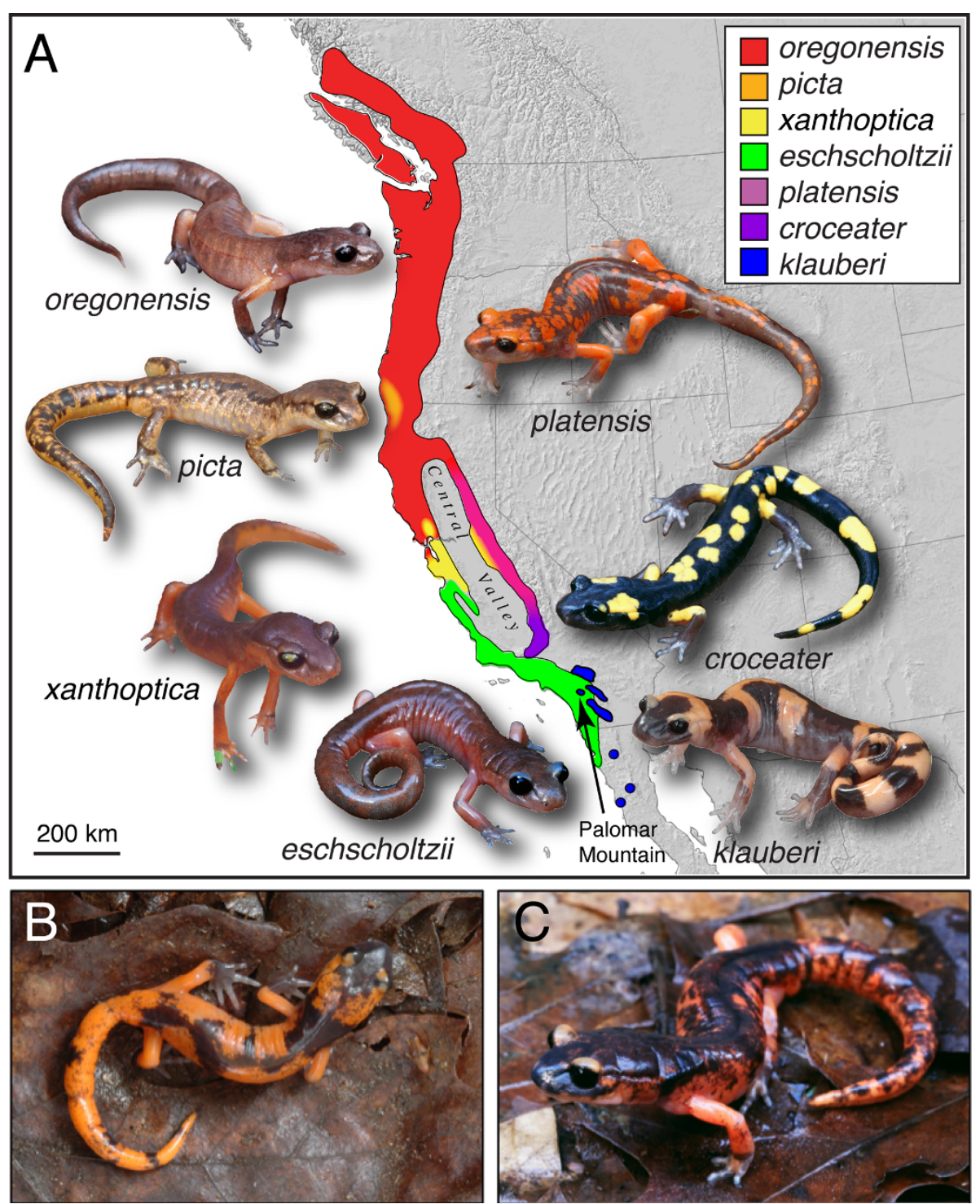

Figure 1 A) Range of the Ensatina eschscholtzii complex and location of the hybrid zone described here (arrow); B-C) hybrid individuals showing intermediate color pattern.

in Ensatina, Stebbins [14] hypothesized that the complex originated in northern California or southern Oregon, where an ancestral range forked into two fronts that expanded southward around the arid Central Valley of California along two separate paths, one along the relatively low-elevation coast ranges, the other inland along the western slopes of the higher-elevation Sierra Nevada. Eventually, the two lineages came back into contact in southern California, creating a ring encircling the Central Valley (Figure 1). Stebbins [14] described broad (up to $150 \mathrm{~km}$ ) intergrade zones where neighboring subspecies met, except where the two ends meet in southern California. Decades of genetic work by D. B. Wake and colleagues using allozymes and mitochondrial
DNA have uncovered remarkably high, geographically structured genetic diversity within Ensatina, suggesting the biogeographic history of Ensatina is much more complex, having featured periods of geographic isolation and multiple instances of secondary contact [13,15-18]. However, these studies are consistent with Stebbins' general biogeographic hypothesis in that 1) genetic distances are higher between the geographically terminal populations than most adjacent populations [13,18], and 2) within subspecies, there is a positive correlation between genetic and geographic distance [16]. Studies of hybridization and introgression within and among subspecies have identified broad zones of introgression among ecologically similar lineages (i.e., within subspecies), but 
abrupt transitions between ecologically divergent subspecies $[13,19]$.

The conclusion that the two geographically terminal subspecies, eschscholtzii and klauberi, are reproductively isolated is central to the ring species interpretation, and also to unresolved debates about species boundaries in this complex [20-23]. Although the sympatry of the terminal forms was not yet known to Stebbins in 1949, it was later discovered that these forms are sympatric in four geographically isolated contact zones in southern California [24-27]. Brown [24] examined color pattern and blood serum proteins from three of these contact zones, including a region on Palomar Mountain covering approximately 10 square miles where several narrow zones of sympatry were found with little evidence of introgression [24]. Marked differences in the habitats occupied by klauberi and eschscholtzii were noted in the Palomar region, with eschscholtzii occupying oak-chaparral associations below $\sim 1,370 \mathrm{~m}$ and klauberi inhabiting pine-oak-cedar forest above this elevation [24]. Hybridization occurred in an ecotone between these two habitat types between $\sim 1,220$ $1,370 \mathrm{~m}$ [24]. Wake et al. [28] typed samples of individuals from all four contact zones at 26 allozyme loci, finding evidence of hybridization (albeit infrequent) at all but the southernmost contact in the Cuyamaca Mountains (see also [13]).

Although these studies provided the first evidence for hybridization between klauberi and eschscholtzii, individuals were sampled at a relatively broad geographic scale, and analyses were limited to identification and classification of hybrid individuals. The present study extends previous work on hybridization at the end of the ring in three ways. First, the geographic scale of this study is much finer than previous work, an important consideration given that hybrid zones may vary in structure (i.e., clinal vs. mosaic) at different sampling resolutions [29-31]. In general, sampling should be undertaken at the scale at which individuals meet, mate, and produce offspring (tens of meters in Ensatina; [32,33]). Second, although habitat isolation has been implicated as a strong barrier to gene flow between sympatric populations of Ensatina [19,24,28], our study is the first to examine whether habitat-genotype associations exist and to formally test the hypothesis that habitat type is structuring the hybrid zone [24]. Finally, this study is the first to use maximum-likelihood analysis of multilocus clines and linkage disequilibrium to make inferences about selection in a hybrid zone at the end of the ring.

A solid theoretical framework exists for studying the evolution and maintenance of clines [34-37]. After any initial contact between differentiated populations, clines in allele frequencies will exist, the scale of which depends on the dispersal scale of the organism, the strength of selection and time since contact, and the shape of which will be determined by asymmetric and epistatic effects
$[2,38]$. If there is neutral mixing, steep gradients in allele frequencies will decay over time resulting in wide, shallow clines; conversely, if there is a strong barrier to gene flow because of selection against hybrids and/or assortative mating, steep clines may be maintained $[2,10,39]$. Selection may act against hybrids independent of geographic location, or along environmental gradients where local adaptation favors one parental form in one habitat, and the other in another [34-36]. In the latter scenario, migrants dispersing into the neighboring habitat keep the taxa mixed and prevent further local adaptation. All of these models assume that dispersal is random with respect to phenotype and the environment. However, it is possible that an individual's decision about whether and where to disperse depends on its fitness in a given environment, with different genotypes moving into preferred habitat patches where they are locally adapted [40,41]. It seems reasonable that such fitness-dependent dispersal may result in fine-scale genotype-habitat associations [42]; when such associations exist, simple hybrid zone models may not effectively describe spatial variation in allele frequencies $[43,44]$, but can be extended to take such associations into account [45]. In addition, even when selection against hybrids/hybridization is not tied to the environment, tension zones resulting from endogenous selection will tend to seek out geographic barriers to gene flow and/or habitat suitability troughs in the environment, where they will become trapped [38]. The interaction of endogenous and exogenous factors where tension zones come to rest may further steepen clines. For these reasons, incorporating information on local environmental variation is important in understanding the balance between local adaptation and dispersal and its effect on hybrid zone structure.

Here, we present a detailed genetic analysis of a hybrid zone on Palomar Mountain between the geographically terminal forms of the Ensatina complex. Our goal is to analyze the barrier to gene flow between these sympatric forms to provide insight into the nature and extent of reproductive isolation between the terminal forms of a ring species. We use two complementary analytical approaches. First, we use individual-based Bayesian methods for identifying and classifying hybrids using multilocus genetic data to estimate levels and direction of hybridization $[46,47]$. We then use maximum-likelihood analysis of linkage disequilibrium and cline shape to make inferences about dispersal and selection in the hybrid zone $[48,49]$. We test for genotype-habitat associations, and whether including this variation improves the fit of clines [cf. 45]. If a strong barrier to gene flow exists between the hybridizing taxa, we would expect to see: 1) concordant clines among independent loci that are narrow with respect to dispersal; 2) deviations from Hardy-Weinberg equilibrium at diagnostic loci due to 
nonrandom mating and/or selection against hybrids; and 3) nonrandom associations (linkage disequilibria) between alleles characteristic of each parental type, due predominantly to dispersal of parental individuals into the hybrid zone [10,48-50]. By examining patterns of cytonuclear disequilibria, we provide insight into possible mechanisms of selection and patterns of mating in the hybrid zone [51] that may be important for maintaining species boundaries. Finally, we discuss the evolutionary implications of introgression and reproductive isolation at the end of the ring for divergence within the Ensatina complex as a whole.

\section{Methods}

\section{Sampling}

The study site is located on Palomar Mountain, San Diego County, California (Figure 1). Suitable habitat consists of mixed montane woodland and montane riparian forest dominated by Incense Cedar (Calocedrus decurrens), White Fir (Abies concolor), Black Oak (Quercus kelloggi), Coast Live Oak (Quercus agrifolia), Bigcone Douglas Fir (Pseudotsuga macrocarpa), and Canyon Live Oak (Quercus chrysolepis). The area sampled is approximately $3.5 \mathrm{~km} \times 1.75 \mathrm{~km}$. The sampling distribution primarily follows a northeast-facing slope and boulder-filled creek adjacent to an open treeless meadow of unsuitable habitat (Figure 2). Salamanders were captured using visual surveys of natural cover objects during the day and night driving. All individuals that were encountered were sampled, and latitude, longitude, and elevation (with error estimates $<10 \mathrm{~m}$ ) were recorded at the point of capture using a GPS (Additional file 1). Three hundred and thirty-five salamanders were sampled over a threeyear period from January-April of 2005-7. Individuals were classified based on diagnostic color pattern as klauberi, eschscholtzii, or hybrids (Additional file 1). Hybrids are readily identified by their aberrant color pattern (Figure 1). Most individuals were sampled non-lethally by removing a piece of the tail tip to allow for future longterm monitoring of the nature of this zone; a subset of individuals were euthanized and preserved as voucher specimens and deposited in the Museum of Vertebrate Zoology (MVZ), University of California, Berkeley. Tissue samples collected in the field were stored in $95 \%$ ethanol or propylene glycol and later frozen at $-80^{\circ} \mathrm{C}$ in the lab. Individuals that were not collected were photographed and marked using subcutaneous alphanumeric tags (Northwest Marine Technology, Shaw Island, WA) and returned to their point of capture within 24 hours. All research procedures using live animals were conducted in accordance with the University of California, Berkeley's institutional animal care and use committee protocol (R278-0410) issued to CM.

\section{Molecular markers}

DNA was extracted from tissues (liver or tail-tip) using Qiagen DNeasy tissue kits following the manufacturer's protocol (Qiagen, Valencia, CA). Using PCR, we amplified three nuclear protein-coding loci (CXCR4, SLC8A3, RAG1) and one mitochondrial locus (ND4) for all 335 individuals. PCRs consisted of 40 cycles of $94^{\circ} \mathrm{C}$ for $30 \mathrm{~s}, \mathrm{Ta}^{\circ} \mathrm{C}$ for $45 \mathrm{~s}$, and $72^{\circ} \mathrm{C}$ for $60 \mathrm{~s}$, with locus specific annealing temperatures (Table 1). Primer sequences are based on primers published elsewhere [52-55]. PCR products were purified using ethanol following standard methods. The mitochondrial PCR product was used in a PCR-RFLP assay with the HindIII restriction endonuclease following the manufacturer's protocol (New England Biolabs, Ipswich, MA). The enzyme recognizes a single restriction site in individuals with klauberi mitochondrial DNA, resulting in a double-band profile for klauberi mtDNA and a singleband profile for eschscholtzii mtDNA. Restriction fragments were visualized on standard agarose gels and scored. Autosomal loci were sequenced in the forward direction only for most individuals. Purified templates were sequenced using dye-labeled dideoxy terminator cycle sequencing. DNA sequences were edited and aligned using Geneious Pro v5.3 [56]. Raw DNA sequence alignments are provided in additional files 2, 3 and 4. Invariant sites and ambiguous positions were removed from the alignments. We resolved haplotypes using PHASE $[57,58]$ after first using SeqPHASE [59] to convert between FASTA and PHASE files.

\section{Identification and classification of hybrids}

Structure $[47,60,61]$ and NewHybrids $[46,62]$ were used to identify and classify hybrids using the multilocus sequence data. In the context of a two-population hybrid zone, Structure jointly assigns individuals probabilistically to the two parental populations $[47,60,61]$, while NewHybrids computes the posterior probability that an individual belongs to distinct genotype frequency classes (e.g., parentals, F1s, F2s, and backcrosses) arising from early generation matings between two species $[46,62]$. We coded each unique haplotype as a different allele (Additional file 5). In Structure, we used the admixture model and assumed two populations with independent allele frequencies $(\lambda=1)$. We ran 100,000 sweeps of five chains after a burn-in of 50,000 sweeps and checked for convergence by comparing the estimated membership coefficient $(Q)$ for each individual across the 5 runs. For NewHybrids, we used the default genotype categories for first- and second-generations of crossing and ran 100,000 sweeps of five chains started from overdispersed starting values after a burn-in period of 50,000 sweeps following the software author's recommendation. Uniform priors were used for the mixing 


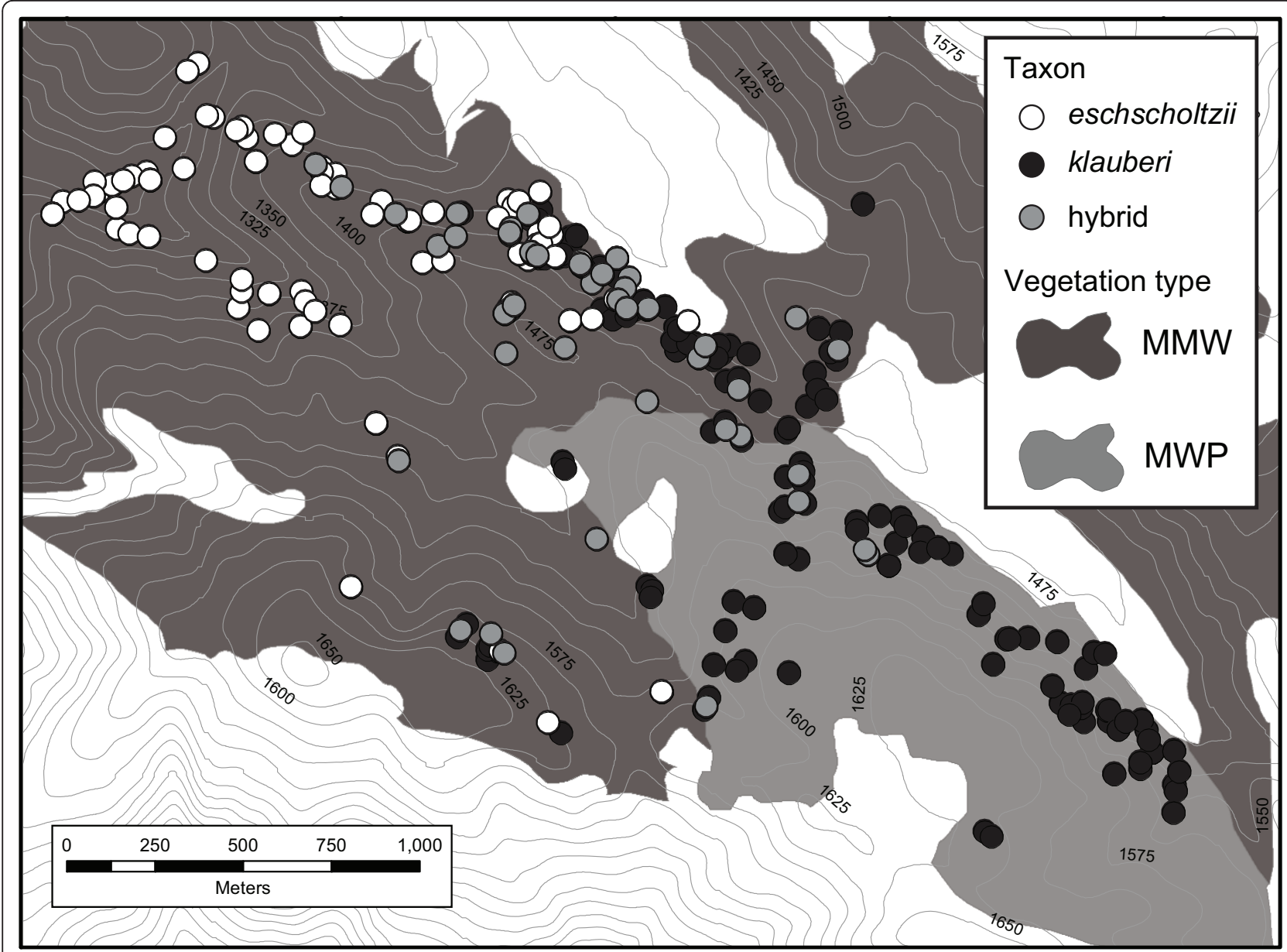

Figure 2 Distribution of klauberi, eschscholtzii, and hybrid individuals across the contact zone (based on phenotype and genotype) Suitable habitat is represented by gray polygons, where MMW = Mixed Montane Woodland and MWP = Mixed Woodland with Bigcone Douglas fir (Pseudotsuga macrocarpa). Vegetation map modified from [70].

proportions and allele frequencies. To check for convergence, we visually inspected $P(z)$ values from the different runs which were then averaged across the 5 runs. A threshold hybrid index ( $Q$-value) was calculated to classify individuals as "hybrids" or "pure parentals" in Structure. For three diagnostic loci (six alleles), there are seven categories (i.e., an individual may contain $0,1,2$,
$3,4,5$, or 6 alleles from taxon 1) and thus "hybrids" were defined as any individual with a $Q$-value between 0.1 [i.e., $(0+1 / 7) / 2$ ] and 0.9 [i.e., $(1+6 / 7) / 2$ ], while any individual with a $Q$-value $<0.1$ or $>0.9$ was considered to be a pure parental. The same threshold was used to distinguish hybrids from pure parentals for the NewHybrids analysis, with posterior probability values summed

Table 1 PCR annealing temperatures, sequence length, and primer sequences

\begin{tabular}{|c|c|c|c|c|}
\hline Locus & $\begin{array}{l}\text { Annealing } \\
\text { temp. }\end{array}$ & $\begin{array}{l}\text { Length/ } \\
\text { \# variable sites (bp) }\end{array}$ & PCR primer & Primer sequence $\left(5^{\prime}-3^{\prime}\right)$ \\
\hline \multirow[t]{2}{*}{$\overline{\mathrm{ND} 4}$} & $48^{\circ} \mathrm{C}$ & RFLP & ND4 & CACCTATGACTACCAAAAGCTCATGTAGAAGC \\
\hline & & & LEU & ACCACGTTTAGGTTCATTTTCATTAC \\
\hline \multirow[t]{2}{*}{ CXCR4 } & $55^{\circ} \mathrm{C}$ & $116 / 4$ & CXCR4F2 & TGGTCTGTGGATGCTGTCAT \\
\hline & & & CXCR4R & TGCAGTAGCAGATCAAGATGA \\
\hline \multirow[t]{2}{*}{ SLC8A3 } & $58^{\circ} \mathrm{C}$ & $342 / 6$ & SLC8A3F & CATTCGGGTCTGGAATGAAA \\
\hline & & & SLC8A3R & ACACCACCATCCCCTCTGTA \\
\hline \multirow[t]{2}{*}{ RAG1 } & $56^{\circ} \mathrm{C}$ & $359 / 7$ & Amp F1 & ACAGGATATGATGARAAGCTTGT \\
\hline & & & RAG1R & TTRGATGTGTAGAGCCAGTGGTGYTT \\
\hline
\end{tabular}


across hybrid classes for an individual [46,62]. Information about the taxon of origin of individuals was not used for either analysis.

\section{Cline analysis}

Phased haplotypes were used to identify allelic states associated with each source taxon (i.e., "eschscholtzii" and "klauberi" alleles) for cline analysis. All loci were diagnostic for one parental form or the other, allowing nearly all haplotypes to be unambiguously assigned. Two haplotypes found in four individuals for one of the loci (SLC8A3) could not be unambiguously assigned and were excluded from the cline analysis. We assumed that allele frequencies at individual loci did not change significantly over the three-year sampling period. Individuals were not pooled into discrete samples for cline fitting.

Sampling sites were collapsed onto a one-dimensional transect using the Pooled Adjacent Violators Algorithm (PAVA) [63], a method for finding the maximum-likelihood (ML) monotonic cline over a set of observations [64]. The advantage of the PAVA method is that it doesn't depend on a particular cline model; it assumes only a monotonic increase or decrease in allele frequencies across sampling sites [64]. Because the path of the contact front is assumed to be linear in the PAVA method, the best-fit axis of the transect orientation through sampled individuals was estimated using a one-dimensional cline fitting with a straight centerline. The best-fit axis of orientation equated to a heading of $207^{\circ}$ (support limits $200^{\circ}, 211^{\circ}$ ). There was no evidence for different orientation among different loci. Monotonic clines and their likelihood profiles were estimated using a routine written in Mathematica [65] by SJEB for Analyse 2.0beta. The width of a cline is usually defined as the inverse of the maximum slope [37]. However, because it is not clear how best to estimate the maximum slope from monotonic clines, we estimated cline widths by fitting parametric sigmoid clines using Analyse 1.3 [66]. A sigmoid cline in allele frequencies $(p)$ can be modeled by a tanh function of its width and center, such that

$$
p=(1+\tanh [2(x-c) / w]) / 2
$$

where $c$ is the cline center, $w$ is the cline width and $(x-c)$ is the distance from the cline center [48]. We fit sigmoid clines and did not explore more complex stepped cline models because doing so only seems justified when there is sufficient sampling in the tails of the cline [38]. Parameter estimates are given as maximum-likelihood estimates, along with two-unit support limits analogous to $95 \%$ confidence intervals [67].

Cline shape concordance among loci was assessed using Barton's concordance analysis [48,68]. In Barton's approach, a hybrid index $(H I)$ is calculated for each individual and scaled from 0 to 1 , expressed as the proportion of alleles derived from one of the two parental types (in this case, klauberi). This set of alleles can be further partitioned into subsets of alleles coming from different loci (e.g., the individual's alleles from locus 1) and a $H I$ calculated for each subset, resulting in individual $\times$ locus HIs [68]. If all loci introgress equally, the expectation for an individual $\times$ locus $H I$ is the same as for an individual's $H I$. Thus, under the expectation for equal introgression across loci, the plot of individual $x$ locus $H I$ s against individual $H I$ s will fall on the diagonal (i.e., $x=y$ ). Deviations from this equal-introgression expectation can be expressed as

$$
H I_{l o c}=H I+H_{e}[\alpha+\beta(2 H I-1)]
$$

where the locus expectation can deviate from $x=y$ as a function of the expected heterozygosity, $H e=2 H I(1$ $H I$ ). The $\alpha$ parameter (directionality) shifts introgression toward one or the other genome (in this case, toward eschscholtzii) and the $\beta$ parameter (abruptness) allows the introgressive change at a locus to be more or less abrupt than the equal-introgression expectation [68].

Barton's concordance method [48] is an individualbased method independent of geographic information. We also fitted geographic clines to each locus separately and assessed cline coincidence using the likelihood method described by Phillips et al. [50]. The likelihood surface of each locus was explored stepwise along axes for both center position $(c)$ and width $(w)$ while allowing other parameters to vary at each point [50]. Likelihood profiles [69] were constructed for both $c$ and $w$ and summed over all loci, resulting in a log-likelihood profile for the ML shared center or width [50]. The shared ML estimate was compared to the sum of noncoincident profile ML estimates using a likelihood ratio test [69]. Twice the difference in $\log$ likelihood $(G=2 \Delta \mathrm{LL})$ between the two models under comparison is significant at level $\alpha$ if $G=2 \Delta \mathrm{LL}>\chi_{\mathrm{df}, \alpha}^{2}$ with the degrees of freedom equal to the difference in the number of parameters between the two models $[48,49]$.

\section{Testing for genotype-habitat associations}

We tested whether hybrids and pure parentals show differences in elevation and vegetation type to see if a simple clinal hybrid zone model was appropriate for describing spatial variation in allele frequency. If significant genotype-habitat associations exist, a simple clinal model may be a poor description of the observations [43] relative to a clinal model that takes such associations into account [45]. We used elevation estimates taken at the point of capture for each individual and vegetation data from a floristic study of Palomar Mountain State Park [70]. Individuals were classified as coming from one of 
two vegetation series, Mixed Montane Woodland (MMW) or Montane Woodland with Pseudotsuga macrocarpa (MWP) (Figure twenty-eight in [70]). These series intergrade [70] and do not have sharply defined borders in and around the hybrid zone, but there is a discernible transition from MMW in the northwestern, lower-elevation portion of the transect to MWP in the southeastern, higher-elevation portion of the transect (Figure 2). Statistical analyses were performed using SPSS Statistics 17.0 (IBM).

To examine whether genotype-habitat associations contributed to the observed spatial structure of the hybrid zone, we compared a model incorporating variation in habitat and elevation (the "habitat-and-cline" model) to one assuming no genotype-habitat associations (the "cline only" model" model) using likelihoodratio tests [cf. 45], accepting the more complex (i.e., more parameter rich) model only when justified by a significant increase in the likelihood of the observations.

\section{Estimating linkage disequilibria}

Average pairwise linkage disequilibrium $(D)$ was estimated in sets of sampled genotypes using the likelihood approach implemented in Analyse 1.3 [66]. Sets of genotypes were formed in a window sliding across the most likely orientation of the hybrid zone using a window size of 200 meters moved in 100 meter increments along the cline using a routine written in Mathematica [65] and implemented in Analyse 2.0beta. $D$ is a measure of the statistical association of allelic states across loci, which ranges from -0.25 through zero to 0.25 . When considering a single diploid locus (rather than two haploid loci), the analog of $D$ is heterozygote deficit [71]. We calculated: 1) within locus disequilibrium (the ML estimate of heterozygote deficit over the three diploid nuclear loci); 2) between nuclear loci disequilibrium (the ML estimate of pairwise $D$ over the three possible pairs of the nuclear loci); and 3) cytonuclear disequilibrium (the ML estimate of pairwise $D$ over the three possible pairs of nuclear/mt markers).

\section{Results}

\section{Hybrid zone genotypes}

There is a clear transition at all loci from eschscholtzii alleles in the northwest to klauberi alleles in the southeast. Hybrids are concentrated near the estimated consensus center of the hybrid zone. Initial identification of individuals based on color pattern as eschscholtzii, klauberi, or hybrid was generally consistent with genetic classification based on Structure and NewHybrids (Additional File 1). Forty-six individuals initially identified as hybrids based on color pattern were classified as such by Structure with a $Q$-value between 0.1 and 0.9 , and by NewHybrids with posterior probability $>0.8$ (Figure $3 \mathrm{~A}$ ). Of the 46 hybrid individuals, 42 possessed klauberi mtDNA (Figure 3B).
Twenty-two of the 46 hybrid individuals were classified as F1s with posterior probabilities $>0.8$ (Figure $3 \mathrm{C}$ ). Where individuals were estimated to be F2s or backcrosses, the estimates were not well-supported (Figure 3C), though these individuals appear to contain more eschscholtzii alleles. Of the four hybrids with eschscholtzii mtDNA, two were classified as F1s ( $p p>0.85$; Figure 3C).

\section{Genotype-habitat associations}

Parental forms are associated with different vegetation series in the contact zone (Chi-square test, $p<0.001$ ). Eschscholtzii are found almost exclusively in the MMW series along with $>80 \%$ of the hybrids, while klauberi individuals are found at roughly equal frequencies in the two vegetation series. Eschscholtzii occupies a broader, but lower elevational range (1200-1601 m; mean $1360 \mathrm{~m}$ ) than klauberi (1297-1694 m; mean $1470 \mathrm{~m}$ ) and hybrids (1342$1601 \mathrm{~m}$; mean $1445 \mathrm{~m}$ ) (One-way ANOVA, Tukey's HSD, $p<0.001$ ). Modification of the underlying clines according to vegetation type [cf. 45] gave no significant improvement in cline fit, and modification according to elevation provided only a slight improvement (results not shown).

\section{Cline shape and concordance}

Cline width and center estimates and the $\alpha$ and $\beta$ components of Barton's [48] concordance analysis are provided in Table 2. Both mitochondrial and nuclear loci change over approximately the same distance (cline widths of 718-799 m; Table 2). The three nuclear loci were concordant (Figure 4B-D). The mitochondrial locus however, was not concordant with the nuclear loci (Figure 4A), showing a shift toward the range of eschscholtzii by approximately $15 \%$ of the consensus cline width (approximately $100 \mathrm{~m}$ ). Maximum-likelihood profiles for the centers and widths of clines are shown in Figure 5. Likelihood-ratio tests showed no significant difference in cline width when all four loci were considered separately versus when they were considered together.

\section{Linkage disequilibria}

Results of the sliding-window analysis of linkage disequilibria are shown in Figure 6. Genetic disequilibrium (including heterozygote deficit and cytonuclear disequilibrium) is concentrated on the eschscholtzii side of the hybrid zone, where within and between nuclear loci estimates of $D$ reach maximum values of approximately 0.15 . Cytonuclear disequilibrium is found exclusively on the eschscholtzii side of the zone.

\section{Discussion}

Earlier work based on allozymes has shown that hybridization is infrequent, or even absent in at least one area of sympatry at the end of the Ensatina ring [27,28]. Our fine-scale analysis revealed a much higher frequency of 


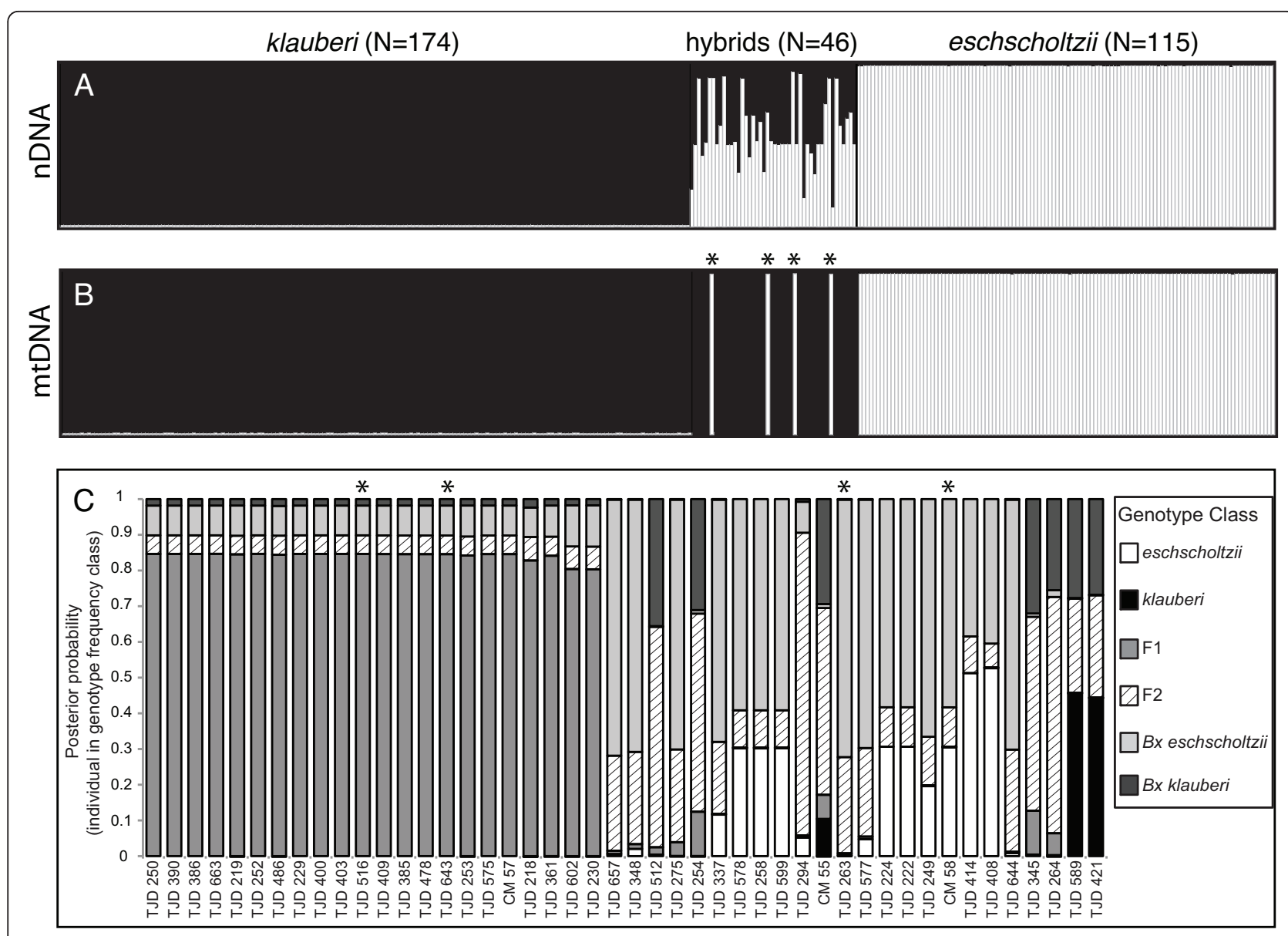

Figure 3 Identification and classification of hybrids. A) Structure results showing proportion of membership from each parental population for all 335 individuals; B) MtDNA haplotypes for all individuals; C) NewHybrids classification of the 46 hybrids classified by genotype frequency class. The four individuals with asterisks in (B) and (C) represent hybrids with eschscholtzii mtDNA; all other hybrids possess klauberi mtDNA.

hybridization compared to previous estimates for Palomar Mountain as well as other contacts between eschscholtzii and klauberi based on allozymes and morphology $[27,28]$. Pure parentals and F1s dominated the sample. The hybrid zone is narrow (cline width estimates of 718-799 m) with respect to per generation dispersal estimates (maximum 120 m; [32]). Although significant genotype-habitat associations exist, modification of the underlying clines according to vegetation type did not improve the fit of clines, and modification by elevation provided only a minor improvement. The predominance of genetic disequilibrium (including heterozygote deficit and cytonuclear disequilibrium) on the eschscholtzii side of the hybrid zone, coupled with the fact that nearly all hybrids have klauberi mtDNA suggests that either: 1) the hybrid zone may be moving (toward the range of eschscholtzii) and/or 2) the zone is static, but gene flow is asymmetric (from klauberi to eschscholtzii). Without

Table 2 Maximum-likelihood estimates (with lower, upper support limits) of cline centers and widths along with the $\alpha$ and $\beta$ components of Barton's concordance analysis $[48,68]$

\begin{tabular}{|c|c|c|c|c|}
\hline Locus & Center & Width (km) & $\alpha$ & $\beta$ \\
\hline All loci & $-0.112(-0.138,-0.087)$ & $0.764(0.704,0.830)$ & - & - \\
\hline ND4 & $-0.202(-0.274,-0.136)$ & $0.718(0.580,0.898)$ & 0.753 & -1.522 \\
\hline All nuclear loci & $-0.097(-0.125,-0.070)$ & $0.765(0.700,0.838)$ & - & - \\
\hline CXCR4 & $-0.091(-0.140,-0.045)$ & $0.770(0.661,0.901)$ & -0.091 & 0.040 \\
\hline SLC8A3 & $-0.113(-0.164,-0.065)$ & $0.799(0.687,0.934)$ & 0.084 & -0.258 \\
\hline RAG1 & $-0.087(-0.134,-0.042)$ & $0.725(0.620,0.851)$ & 0.026 & 0.248 \\
\hline
\end{tabular}

Center and width units are in kilometers and centers are measured with the gnomonic projection focus at zero. 

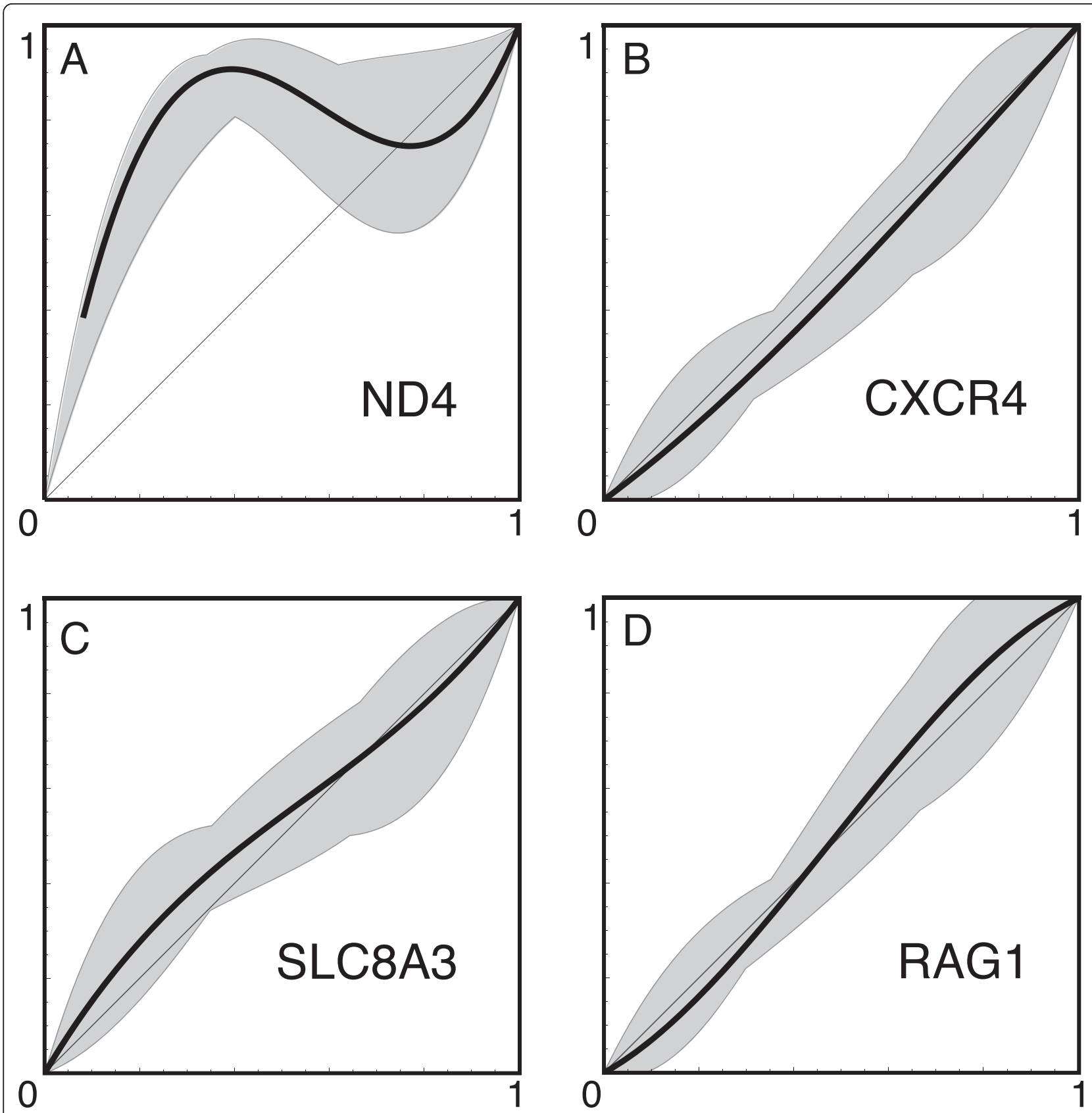

Figure 4 Comparison of mitochondrial (A) and nuclear (B-D) cline concordance using Barton's concordance analysis. Plots show the individual per-locus hybrid index plotted against the hybrid index calculated over all loci. The equal-introgression expectation lies on the diagonal. Per locus fits can deviate toward either genetic background $(\alpha)$ and by having more or less abrupt change ( $\beta$ ) (see text). The nuclear clines are concordant with each other (and the consensus nuclear cline), while the mitochondrial cline is not; individuals are more likely to have klauberi $\mathrm{mtDNA}$ than expected under equal introgression across loci $(\alpha=0.753)$.

long-term data on the spatiotemporal dynamics of the zone, these hypotheses are difficult to disentangle [10]. Barton and Hewitt [10] argued that most hybrid zones are clines maintained by a balance between dispersal and selection against hybrids ("tension zones" [72]), and can move from place to place because they are not maintained by local environmental conditions. Tension zone movement may be caused by differences in fitness, 

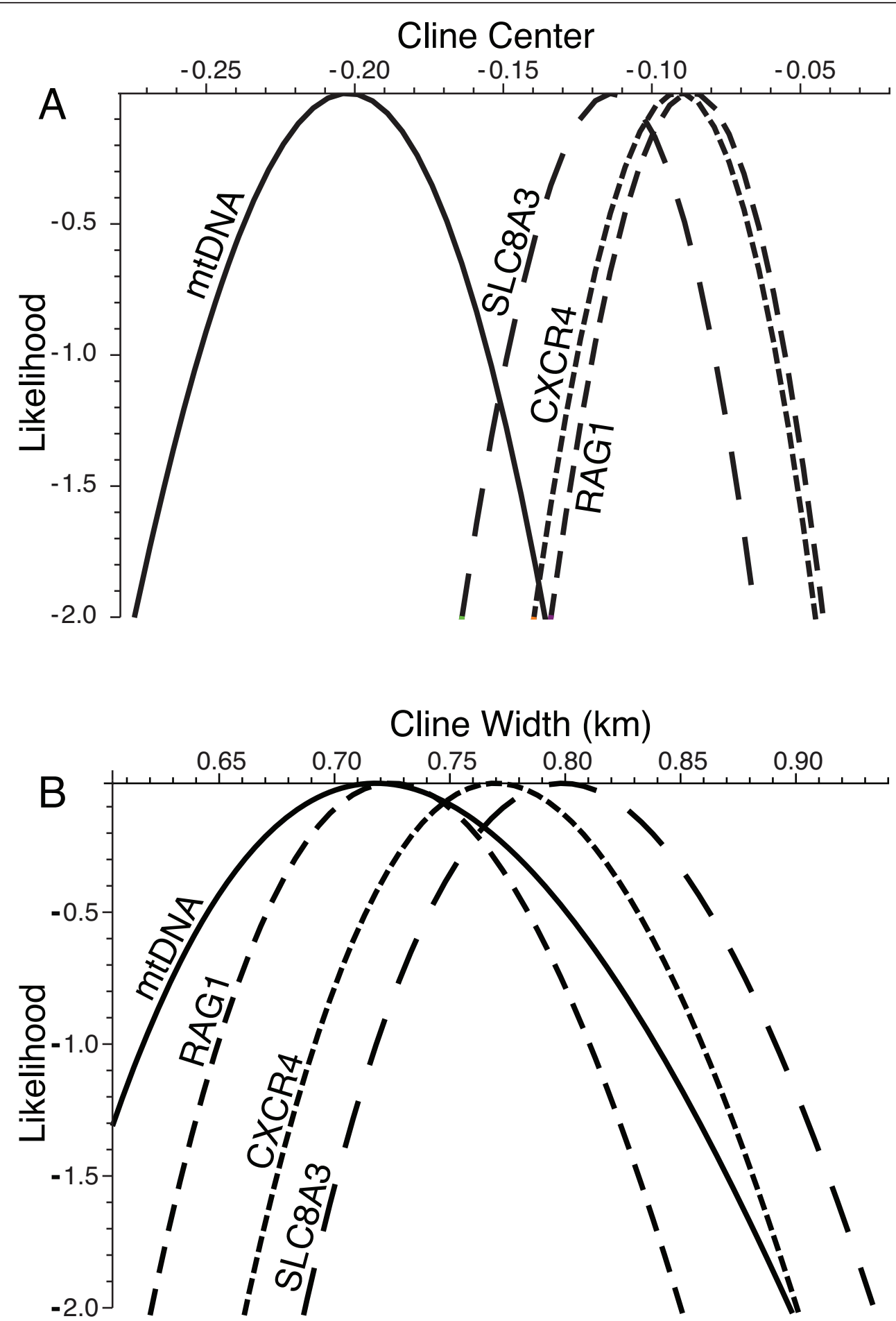

Figure 5 Maximum likelihood profiles for the centers (A) and widths (B) of clines. Cline centers for the nuclear loci are concordant with each other, while the mitochondrial cline is shifted significantly to the west (A). There is no significant difference in cline widths (B).

density, dispersal, or hybridization asymmetry between parental forms $[2,10,73,74]$. For this reason, although they are not maintained by the local environment, tension zones will move toward and become associated with barriers to gene exchange, including environmental factors that reduce density or dispersal [10]. Our observations are consistent with a tension zone trapped at an ecotone, with gene combinations characteristic of 


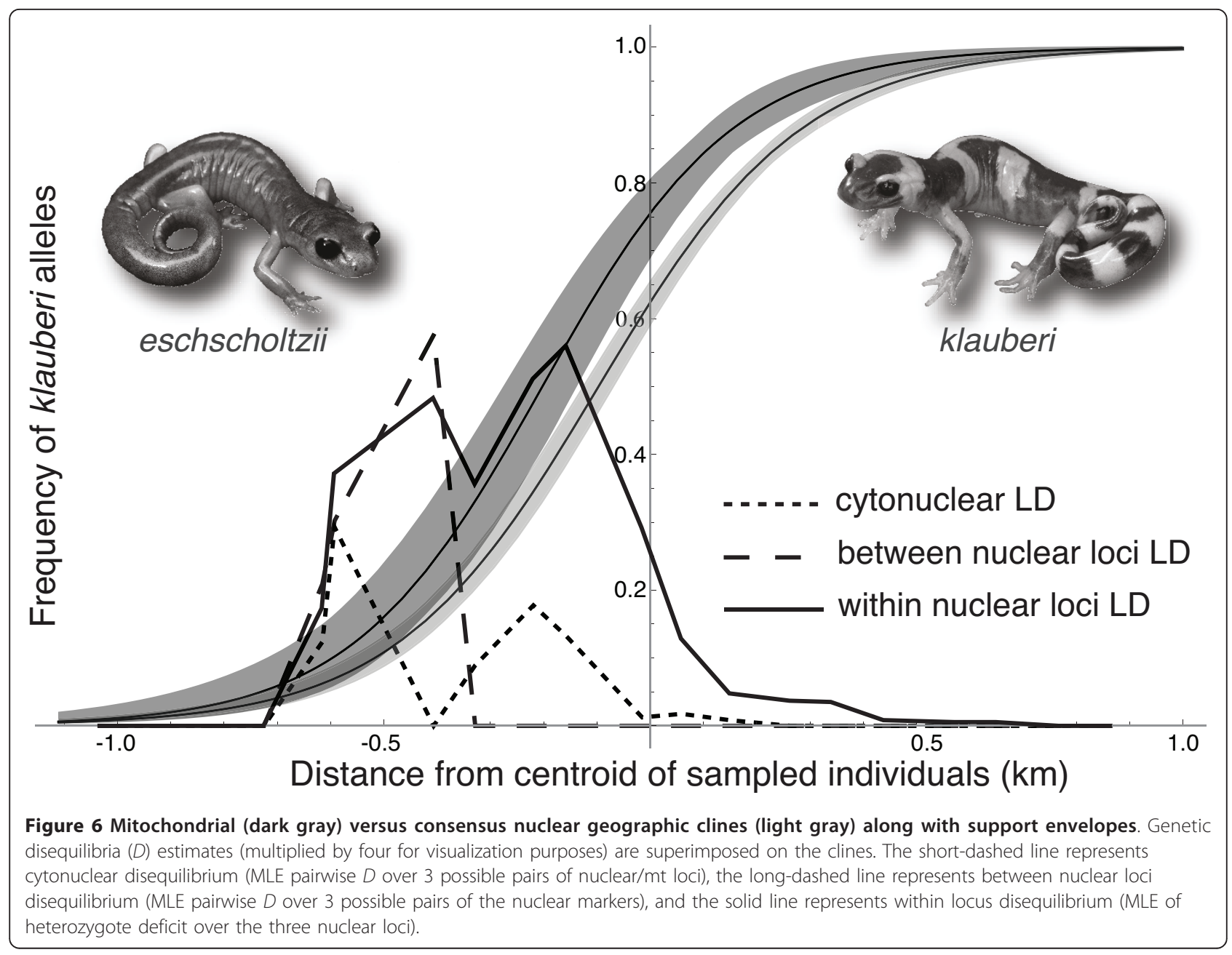

klauberi showing up on the eschscholtzii side of the zone due to asymmetric hybridization, giving a strong signal of genetic disequilibria. Given that nearly all of the hybrids possess klauberi mtDNA, either: 1) hybridization between eschscholtzii and klauberi is (mostly) unidirectional, with F1 hybrids formed from female klauberi mating with male eschscholtzii (but not vice versa), implying asymmetric prezygotic isolation, or 2) hybridization is reciprocal, but offspring resulting from female eschscholtzii mating with male klauberi (or male hybrids) are inviable (implying asymmetric postzygotic isolation [75]). Hybrids often possess the mitochondrial DNA of only one of two parental species in nature [76-78], a pattern predicted in species with female-choice when females of a rare species are unable to find conspecific males because they are scarce, and eventually accept matings with heterospecific males of a more common species [79]. However, the two taxa appear to be equally abundant in zones of overlap at Palomar Mountain, suggesting that factors other than rarity of conspecific klauberi males are responsible for the disproportionate percentage of hybrids with klauberi mtDNA. The presence of four hybrid individuals with eschscholtzii mtDNA suggests matings between female eschscholtzii and male klauberi (or male hybrids) are possible, but appear to be rare, or that the offspring usually do not survive. Like many animals, female Ensatina are the choosier sex because they invest relatively more than males in reproduction, and are courted by promiscuous males, both of their own and closely related species $[1,80]$. If there are costs associated with heterospecific matings (e.g., because hybrids are less fit, or, because these matings produce fewer offspring), there may be strong selection acting on female mating preferences toward increased ability to discriminate between conspecific and heterospecific males [81-83]. This in turn could drive indirect selection on males to track female mating preferences [84].

It is instructive to compare the nature of hybridization between the terminal forms to that between another pair of morphologically and genetically distinct coastal and inland lineages of Ensatina - xanthoptica and platensis, respectively - in the foothills of the central Sierra Nevada 
$[19,24,28]$. These morphological analogs of eschscholtzii and klauberi are thought to have come into contact at some point during the Pleistocene when climate in California was cooler and wetter, allowing xanthoptica to cross the now arid Central Valley and invade the Sierras from the San Francisco Bay area [14]. Hybrids are abundant in the Sierran contact zones, but few, if any, F1s have been detected [19], in contrast to our results. Alexandrino et al. [19] suggested that reduced opportunities for heterospecific encounters due to habitat preference and/or stronger selection against F1s compared to later generation hybrids could explain this pattern. They estimated cline widths comparable to, or wider than those observed here (730-2000 m), and inferred strong selection against hybridization (46-75\%). For comparison, selection against hybridization has been estimated at $32 \%$ for distinct lineages of lizards of the Sceloporus grammicus complex $[85,86]$ and $17-22 \%$ for the toads Bombina bombina and $B$. variegata $[48,49]$. Although it is tempting to try and estimate selection against hybridization in the contact zone studied here, because the hybrid zone is dynamic and/or asymmetric, we cannot sensibly do so under a standard tension zone model, which assumes a symmetric, static hybrid zone. Nonetheless, it is clear that overall selection against hybrids/hybridization is strong in both cases, yet the two contact zones differ markedly in the frequency of different hybrid genotype frequency classes.

\section{Conclusions}

Geographic variation in hybridization frequency among the four contact zones at the end of the ring $[27,28]$ suggests that reproductive isolation may not be uniform across contact zones, perhaps due to differences in local ecological conditions that limit the opportunity for hybridization [see 87], and/or different outcomes of reinforcement $[88,89]$ driven by selection against hybrids owing to the spatial structure of the hybrid zone $[43,90,91]$. This variation provides a rare opportunity to investigate variation in the strength of reproductive isolating barriers within a single species pair in nature, while controlling for among-taxa variation in age, degree of differentiation, strength of postzygotic isolation, etc. [see 1, 87, 92].

The narrow width, shape, and overall concordance of clines for presumably unlinked loci suggest a strong barrier to gene flow between eschscholtzii and klauberi on Palomar Mountain. Introgression of neutral alleles will be delayed, but favorable alleles could quickly cross if prezygotic isolation is weak or absent $[93,94]$. The particular markers themselves probably do not have a direct effect on fitness, but rather they are in linkage disequilibrium with other loci that are under selection. If the amount of linkage disequilibrium that connects selection against hybrids (or hybridization) with an evolving prezygotic isolating mechanism is sufficiently high, then premating isolation may evolve through reinforcement [95]. Future work incorporating information about patterns of mating and gamete utilization will be critical for understanding the role of selection in generating and maintaining species boundaries at the end of the ring $[83,96,97]$.

\section{Additional material}

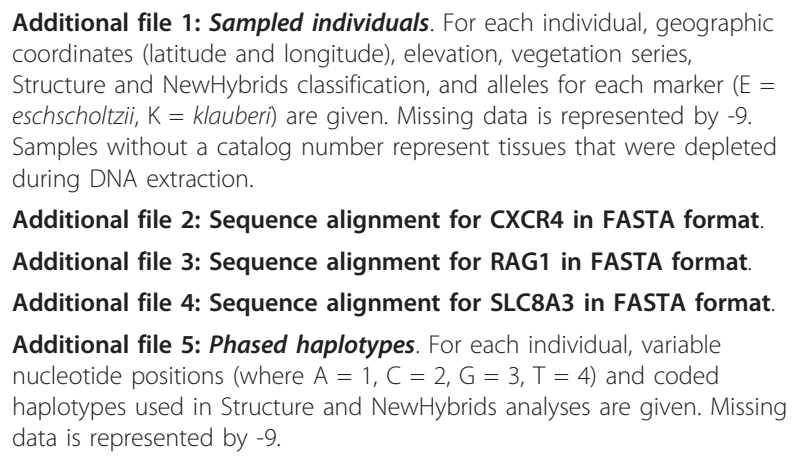

Additional file 1: Sampled individuals. For each individual, geographic coordinates (latitude and longitude), elevation, vegetation series, Structure and NewHybrids classification, and alleles for each marker ( $E$ = eschscholtzii, $\mathrm{K}=$ klauberi) are given. Missing data is represented by -9 . Samples without a catalog number represent tissues that were depleted during DNA extraction.

Additional file 2: Sequence alignment for CXCR4 in FASTA format. Additional file 3: Sequence alignment for RAG1 in FASTA format. Additional file 4: Sequence alignment for SLC8A3 in FASTA format. Additional file 5: Phased haplotypes. For each individual, variable nucleotide positions (where $A=1, C=2, G=3, T=4$ ) and coded haplotypes used in Structure and NewHybrids analyses are given. Missing data is represented by -9 .

\section{Acknowledgements}

We thank Jimmy A. McGuire, David B. Wake, and George K. Roderick for comments on an earlier draft of this manuscript. Eric Anderson kindly provided an OSX version of NewHybrids and guidance with analyses. We thank R. K. Lauri for providing an electronic copy of his unpublished thesis and vegetation map of Palomar Mountain State Park and Michelle Koo for assistance digitizing the map. We are grateful to Susan Cameron for help with vegetation analysis. We thank the ESRI Conservation Program for providing ArcGIS software for our use. For help in the field, we thank Mike Anguiano, Rayna Bell, Chuck Brown, Susan Cameron, Jessica Castillo, Becky Chong, Erin Conlisk, Brandon Endo, Matt Fujita, Emilio Gabbai-Saldate, Zach Hanna, Kory Heiken, Jasmine Junge, Megan Lahti, Ben Lowe, Matt McElroy, Greg Pauly, Ricardo Pereira, Tod Reeder, Mark Roll, Sean Rovito, Kevin Rowe, Frank Santana, Sean Schoville, Sonal Singhal, Tate Tunstall, and John Wiens. We especially thank Kim and Donna Rosier, Bill Stephenson, and the rest of the staff of the Palomar Mountain Christian Conference Center for their hospitality and access to their property. Permission to work in Palomar Mountain State Park was granted by the State of California Department of Parks and Recreation; we thank Mark Jorgensen, Nedra Martinez, and Jeff Lee for access to the park. This work was conducted under a scientific collecting permit issued by the California Department of Fish and Game (SC007654). We thank Kevin Fleming and Art Fong (CDF\&G) for assistance with permitting. Funding for this work was provided by Sigma Xi, the National Science Foundation (Doctoral Dissertation Improvement Grant DEB-0909821 to TJD and NSF DEB-0641078 to CM), the University of California Department of Integrative Biology, and the Museum of Vertebrate Zoology Martens and Louise Kellogg funds.

\section{Author details}

${ }^{1}$ Museum of Vertebrate Zoology and Department of Integrative Biology, 3101 Valley Life Sciences Building, University of California, Berkeley, CA, USA 94720-3160. ${ }^{2} \mathrm{CIBIO}$, University of Porto, Campus Agrário de Vairão, Rua Padre Armando Quintas 7, 4485-661 Vairão, Portugal. ${ }^{3}$ Departamento de Zoología, Instituto de Biología, Universidad Nacional Autónoma de México, Circuito exterior s/n, Ciudad Universitaria, Copilco, Coyoacán, A.P. 70-153/70-233 México, Distrito Federal. C.P. 04510.

\section{Authors' contributions}

TJD designed the study, collected the material and data, performed the Bayesian analyses, interpreted results, and wrote the manuscript. SJEB performed the cline and genetic disequilibria analyses, interpreted results, and provided extensive feedback that greatly improved the manuscript. CM 
provided logistical support and significant intellectual contributions that improved the study and final manuscript. All authors read and approved the final manuscript.

Received: 24 March 2011 Accepted: 22 August 2011

Published: 22 August 2011

\section{References}

1. Coyne JA, Orr HA: Speciation. Sunderland, MA: Sinauer Associates, Inc.; 2004.

2. Endler JA: Geographic variation, speciation, and clines. Princeton, N. J.: Princeton University Press; 1977.

3. Schluter D: Evidence for ecological speciation and its alternative. Science 2009, 323(5915):737-741

4. Cain AJ: Animal Species and Their Evolution. London: Hutchinson House; 1954.

5. Dobzhansky T: Species after Darwin. In A Century of Darwin. Edited by: Barnett SA. London: Heinemann; 1958:19-55.

6. Jordan DS: The origin of species through isolation. Science 1905 22:545-562.

7. Mayr E: Systematics and the Origin of Species. New York: Dover Publications; 1942.

8. Irwin DE, Irwin JH: Circular overlaps: rare demonstrations of speciation. The Auk 2002, 119:596-602.

9. Irwin DE, Irwin JH, Price TD: Ring species as bridges between microevolution and speciation. Genetica 2001, 112-113:223-243.

10. Barton NH, Hewitt GM: Analysis of hybrid zones. Annu Rev Ecol Syst 1985, 16:113-148.

11. Turelli M, Barton NH, Coyne JA: Theory and speciation. Trends Ecol Evol 2001, 16(7):330-343.

12. Mayr E: Animal Species and Evolution: Harvard University Press; 1963.

13. Pereira R, Wake DB: Genetic leakage after adaptive and non-adaptive divergence in the Ensatina eschscholtzii ring species. Evolution 2009, 63(9):2288-2301

14. Stebbins RC: Speciation in Salamanders of the Plethodontid Genus Ensatina. Univ Calif Publ Zool 1949, 54:47-124.

15. Wake DB, Yanev KP: Geographic variation in allozymes in a "ring species", the plethodontid salamander Ensatina eschscholtzii of western North America. Evolution 1986, 40:702-715.

16. Jackman TR, Wake DB: Evolutionary and historical analysis of protein variation in the blotched forms of salamanders of the Ensatina complex (Amphibia: Plethodontidae). Evolution 1994, 48:876-897.

17. Kuchta SR, Parks DS, Mueller RL, Wake DB: Closing the ring: historical biogeography of the salamander ring species Ensatina eschscholtzii. J Biogeogr 2009, 36:982-995.

18. Moritz C, Schneider C, Wake DB: Evolutionary relationships within the Ensatina eschscholtzii complex confirm the ring species interpretation. Syst Biol 1992, 41:273-291.

19. Alexandrino J, Baird SJE, Lawson L, Macey JR, Moritz C, Wake DB: Strong selection against hybrids at a hybrid zone in the Ensatina ring species complex and its evolutionary implications. Evolution 2005, 59(6):1334-1347

20. Highton R: Is Ensatina eschscholtzii a ring-species? Herpetologica 1998, 54:254-278.

21. Wake DB, Schneider C: Taxonomy of the plethodontid salamander genus Ensatina. Herpetologica 1998, 54:279-298.

22. Graybeal A: Naming species. Syst Biol 1995, 44:237-250.

23. Frost DR, Hillis DM: Species in concept and practice: herpetological applications. Herpetologica 1990, 6(87-104).

24. Brown CW: Hybridization among the subspecies of the plethodontid salamander Ensatina eschscholtzii. Univ Calif Publ Zool 1974, 98:1-57.

25. Brown CW, Stebbins RC: Evidence for hybridization between the blotched and unblotched subspecies of the salamander Ensatina eschscholtzii. Evolution 1964, 18(4):706-707.

26. Stebbins RC: Intraspecific sympatry in the lungless salamander Ensatina eschscholtzii. Evolution 1957, 11:265-270.

27. Wake DB, Yanev KP, Brown CW: Intraspecific sympatry in a ring species, the plethodontid salamander Ensatina eschscholtzii, in southern California. Evolution 1986, 40(4):866-868

28. Wake DB, Yanev KP, Frelow MM: Sympatry and hybridization in a "ring species": the Plethodontid salamander Ensatina eschscholtzii. In Speciation and its Consequences. Edited by: Otte D, Endler JA. Sunderland, MA: Sinauer Associates, Inc.; 1989:134-157.

29. Harrison RG: Pattern and process in a narrow hybrid zone. Heredity 1986 56:337-349.

30. Harrison RG, Arnold J: A narrow hybrid zone between closely related cricket species. Evolution 1982, 36:535-552.

31. Harrison RG, Bogdanowicz SM: Patterns of variation and linkage disequilibrium in a field cricket hybrid zone. Evolution 1997, 51(2):493-505.

32. Staub NL, Brown CW, Wake DB: Patterns of growth and movements in a population of Ensatina eschscholtzii platensis (Caudata: Plethodontidae) in the Sierra Nevada, California. J Herpetol 1995, 29:593-599.

33. Stebbins RC: Natural history of the salamanders of the plethodontid genus Ensatina. Univ Calif Publ Zool 1954, 54:47-124.

34. Fisher RA: Gene frequencies in a cline determined by selection and diffusion. Biometrics 1950, 6:353-361.

35. Haldane JBS: The theory of a cline. Journal of Genetics 1948, 48:277-284

36. May RM, Endler JA, McMurtrie RE: Gene frequency clines in the presence of selection opposed by gene flow. Am Nat 1975, 109:659-676.

37. Slatkin M: Gene flow and selection in a cline. Genetics 1973, 75:733-756.

38. Barton NH, Gale K: Genetic analysis of hybrid zones. In Hybrid Zones and the Evolutionary Process. Edited by: Harrison RG. Oxford: Oxford University Press; 1993:13-45.

39. Kruuk LEB, Baird SJE, Gale KS, Barton NH: A comparison of multilocus clines maintained by environmental adaptation or by selection against hybrids. Genetics 1999, 153(4):1959-1971.

40. Armsworth PR, Roughgarden JE: The structure of clines with fitnessdependent dispersal. Am Nat 2008, 172(5):648-657.

41. Edelaar P, Siepielski AM, Clobert J: Matching habitat choice causes directed gene flow: a neglected dimension in evolution and ecology. Evolution 2008, 62:2462-2472.

42. MacCallum CJ, Nürnberger B, Barton NH, Szymura JM: Habitat preference in the Bombina hybrid zone in Croatia. Evolution 1998, 52(1):227-239.

43. Harrison RG, Rand DM: Mosaic hybrid zones and the nature of species boundaries. In Speciation and Its Consequences. Edited by: Otte D, Endler JA. Sunderland, MA: Sinauer Associates; 1989:111-133.

44. Howard DJ: A zone of overlap and hybridization between two ground cricket species. Evolution 1986, 40:34-43.

45. Bridle JR, Baird SJE, Butlin RK: Spatial structure and habitat variation in a grasshopper hybrid zone. Evolution 2001, 55:1832-1843.

46. Anderson EC, Thompson EA: A model-based method for identifying species hybrids using multilocus genetic data. Genetics 2002, 160:1217-1229.

47. Pritchard JK, Stephens M, Donnelly P: Inference of population structure using multilocus genotype data. Genetics 2000, 155:945-959.

48. Szymura JM, Barton NH: Genetic analysis of a hybrid zone between the fire-bellied toads, Bombina bombina and B. variegata, near Cracow in southern Poland. Evolution 1986, 40(6):1141-1159.

49. Szymura JM, Barton NH: The genetic structure of the hybrid zone between the fire-bellied toads Bombina bombina and B. variegata: Comparisons between transects and between loci. Evolution 1991, 45(2):237-261

50. Phillips BL, Baird SJE, Moritz C: When vicars meet: A narrow contact zone between phylogeographic lineages of the rainforest skink, Carlia rubrigularis. Evolution 2004, 58:1536-1548.

51. Arnold J: Cytonuclear disequilibria in hybrid zones. Annu Rev Ecol Syst 1993, 24:521-524.

52. Arévalo E, Davis SK, Sites JW Jr: Mitochondrial DNA sequence divergence and phylogenetic relationships among eight chromosome races of the Sceloporus grammicus complex (Phrynosomatidae) in central Mexico. Syst Biol 1994, 43:387-418.

53. Biju SD, Bossuyt F: New frog family from India reveals an ancient biogeographical link with the Seychelles. Nature 2003, 425:711-714.

54. Roelants K, Bossuyt F: Archaeobatrachian paraphyly and Pangaean diversification of crown-group frogs. Syst Biol 2005, 54(1):111-126.

55. Bossuyt $F$, Milinkovitch MC: Convergent adaptive radiations in Madagascan and Asian ranid frogs reveal covariation between larval and adult traits. Proceedings of the National Academy of Sciences 2000, 97(12):6585-6590

56. Drummond AJ, Ashton B, Cheung M, Heled J, Kearse M, Moir R, StonesHavas S, Thierer T, Wilson A: Geneious., v4.6 2009. 
57. Stephens M, Donnelly P: A comparison of Bayesian methods for haplotype reconstruction from population genotype data. Am J Hum Genet 2003, 73(5):1162-1169.

58. Stephens M, Smith N, Donnelly P: A new statistical method for haplotype reconstruction from population data. Am J Hum Genet 2001, 68:978-989.

59. Flot J-F: SEQPHASE: a web tool for interconverting phase input/output files and fasta sequence alignments. Mol Ecol Res 2010, 10:162-166.

60. Falush D, Stephens M, Pritchard JK: Inference of population structure using multilocus genotype data: linked loci and correlated allele frequencies. Genetics 2003, 164:1567-1587.

61. Falush D, Stephens M, Pritchard JK: Inference of population structure using multilocus genotype data: dominant markers and null alleles. $\mathrm{Mol}$ Ecol Notes 2007, 7(4):574-578.

62. Anderson EC: Bayesian inference of species hybrids using multilocus dominant genetic markers. Philos Trans R Soc Lond, Ser B: Biol Sci 2008, 363:2841-2850.

63. Brunk HD: Maximum likelihood estimates of monotone parameters. Ann Math Stat 1955, 26:607-616.

64. Macholán M, Baird SJE, Munclinger P, Dufková P, Bímova B, Piálek J: Genetic conflict outweighs heterogametic incompatibility in the mouse hybrid zone? BMC Evol Biol 2008, 8(1):271.

65. Wolfram Research, Inc. Mathematica, Version 7.0. Champaign, IL 2008

66. Barton NH, Baird SJE: Analyse - An application for analyzing hybrid zones. Edinburgh: Freeware; 1.31998.

67. Edwards A: Likelihood. Baltimore, MD: Johns Hopkins University Press; 1992.

68. Macholán M, Baird SJE, Dufková $P$, Munclinger P, Vošlajerová Bímová B, Piálek J: Assessing multilocus introgression patterns: A case study on the mouse $\times$ chromosome in Central Europe. Evolution 2011, no-no.

69. Hilborn R, Mangel M: The ecological detective: confronting models with data. Princeton, NJ: Princeton University Press; 1997.

70. Lauri RK: A Comparative Floristic Study of Palomar Mountain State Park. Master of Science. San Diego: San Diego State University; 2004.

71. Kirkpatrick M, Ravigné V: Speciation by natural and sexual selection: models and experiments. Am Nat 2002, 159:S22-S35.

72. Key KHL: The concept of stasipatric speciation. Systematic Zoology 1968, 17:14-22.

73. Barton NH: The dynamics of hybrid zones. Heredity 1979, 43(3):341-359.

74. Hewitt GM: A sex-chromosome hybrid zone in the grasshopper Podisma pedestris (Orthoptera: Acrididae). Heredity 1975, 35:375-387.

75. Turelli M, Moyle LC: Asymmetric postmating isolation: Darwin's corollary to Haldane's rule. Genetics 2007, 176(2):1059-1088.

76. Avise JA, Saunders NC: Hybridization and introgression among species of sunfish (Lepomis): analysis by mitochondrial DNA and allozyme markers. Genetics 1984, 108:237-255.

77. Lushai G, Allen JA, Goulson D, MacLean N, Smith DAS: The butterfly Danaus chrysippus (L.) in East Africa comprises polyphyletic, sympatric lineages that are, despite behavioural isolation, driven to hybridization by female-biased sex ratios. Biol J Linn Soc 2005, 86(1):117-131.

78. Randler C: Avian hybridization, mixed pairing and female choice. Anim Behav 2002, 63:103-119.

79. Wirtz P: Mother species-father species: unidirectional hybridization in animals with female choice. Anim Behav 1999, 58(1):1-12

80. Andersson M: Sexual Selection. Princeton: Princeton University Press; 1994

81. Ehrman L, Wasserman M: The Significance of Asymmetrical Sexual Isolation.Edited by: Hecht MK, Wallace B, Prance GT. Evolutionary Biology. New York: Plenum Press; 1987:21:1-20.

82. Kaneshiro KY, Giddings LV: The Significance of Asymmetrical Sexual Isolation and the Formation of New Species.Edited by: Hecht MK, Wallace B, Prance GT. Evolutionary Biology. New York: Plenum Press; 1987:21:29-43.

83. McPeek MA, Gavrilets S: The evolution of female mating preferences: Differentiation from species with promiscuous males can promote speciation. Evolution 2006, 1967-1980.

84. Lande R: Models of speciation by sexual selection on polygenic traits. Proc Natl Acad Sci USA 1981, 78(6):3721-3725.

85. Marshall JC, Sites JW: A comparison of nuclear and mitochondrial cline shapes in a hybrid zone in the Sceloporus grammicus complex (Squamata: Phrynosomatidae). Mol Ecol 2001, 10:435-449.

86. Sites JW Jr, Barton NH, Reed KM: The genetic structure of a hybrid zone between two chromosome races of the Sceloporus grammicus complex (Sauria, Phrynosomatidae) in Central Mexico. Evolution 1995, 49(1):9-36.
87. Aldridge G: Variation in frequency of hybrids and spatial structure among Ipomopsis (Polemoniaceae) contact sites. New Phytol 2005, 167(1):279-288.

88. Dobzhansky T: Genetics and the Origin of Species. New York: Columbia University Press; 1937.

89. Servedio MR, Noor MAF: The role of reinforcement in speciation: theory and data. Annu Rev Ecol Syst 2003, 34:339-364.

90. Cain ML, Andreasen V, Howard DJ: Reinforcing selection is effective under a relatively broad set of conditions in a mosaic hybrid zone. Evolution 1999, 53(5):1343-1353.

91. Hoskin CJ, Higgie M, McDonald KR, Moritz C: Reinforcement drives rapid allopatric speciation. Nature 2005, 437:1353-1356.

92. Aldridge G, Campbell DR: Variation in pollinator preference between two Ipomopsis contact sites that differ in hybridization rate. Evolution 2007, 61(1):99-110.

93. Barton NH: Genetic hitchhiking. Philos Trans R Soc Lond, Ser B: Biol Sci 2000, 355(1403):1553-1562

94. Pialek J, Barton NH: The spread of an advantageous allele across a barrier: The effects of random drift and selection against heterozygotes. Genetics 1997, 145(2):493-504.

95. Servedio MR: The role of linkage disequilibrium in the evolution of premating isolation. Heredity 2009, 102(1):51-56.

96. Lorch PD, Servedio MR: The evolution of conspecific gamete precedence and its effect on reinforcement. J Evol Biol 2007, 20(3):937-949.

97. Marshall JL, Arnold ML, Howard DJ: Reinforcement: the road not taken. Trends Ecol Evol 2002, 17(12):558-563.

doi:10.1186/1471-2148-11-245

Cite this article as: Devitt et al:: Asymmetric reproductive isolation between terminal forms of the salamander ring species Ensatina eschscholtzii revealed by fine-scale genetic analysis of a hybrid zone. BMC Evolutionary Biology 2011 11:245.

\section{Submit your next manuscript to BioMed Central and take full advantage of:}

- Convenient online submission

- Thorough peer review

- No space constraints or color figure charges

- Immediate publication on acceptance

- Inclusion in PubMed, CAS, Scopus and Google Scholar

- Research which is freely available for redistribution 